\title{
Keywords
}

Cervical insufficiency; Cervical remodeling; Cervical ripening; Spontaneous preterm birth;

Cervical microstructural assessment

\section{The role of the cervix in pregnancy}

In normal pregnancy, despite progressive softening, the cervix keeps the uterus closed until term, and then softens/dilates further to allow for delivery of the fetus. Within minutes after birth, this remarkable structure reconstitutes to close the uterus. The entire complex process

\footnotetext{
"Corresponding author. Address: Maternal-Fetal Medicine, Intermountain Healthcare, Utah Valley Regional Medical Center, 1034 N 500 W, Provo, UT 84604, USA. Tel.: +1 435-655-1348. hfeltovich@ gmail.com (H. Feltovich).

Conflict of interest statement

None declared.

Publisher's Disclaimer: This is a PDF file of an unedited manuscript that has been accepted for publication. As a service to our customers we are providing this early version of the manuscript. The manuscript will undergo copyediting, typesetting, and review of the resulting proof before it is published in its final citable form. Please note that during the production process errors may be discovered which could affect the content, and all legal disclaimers that apply to the journal pertain.
} 
involves an elegant concert of molecular and microstructural events, including precisely timed activation of biochemical pathways and interactions between resident and immune cells and the extracellular matrix (ECM). Our understanding of this multifaceted process is rapidly changing as an explosion of molecular and microstructural information is adding to the body of knowledge about cervical remodeling in pregnancy. Below we provide a brief overview of cervical tissue architecture and cervical remodeling, as well as an introduction to emerging concepts in this rapidly growing field.

\section{Cervical tissue architecture}

The prevailing viewpoint is that the cervix is (i) relatively homogeneous, (ii) composed mainly of ECM/collagen (90\%) with minimal cellular content (10-15\%; e.g. fibroblasts, smooth muscle cells, glandular cells, vascular cells and immune cells), and (iii) structurally and physiologically distinct from the uterus [1,2]. However, recent investigation is rapidly expanding our understanding of human cervical tissue architecture.

Cervical tissue ECM contains proteins (mostly collagen, some elastin) and proteoglycans (e.g. hyaluronic acid and decorin) that serve as a scaffold and govern biochemical and mechanical tissue properties such as strength and elasticity [2,3]. In the 1970s, studies postulated that the cervical collagen network in the ECM consists of three relatively homogeneous and distinct zones: inner and outer zones of collagen fibers oriented parallel to the endocervical canal (theoretically to prevent the cervix from tearing off the uterus during dilation), and a circumferential middle layer that may serve as a ratchet to control cervical dilation (Fig. 1) [4,5]. Although this theory remains generally relevant, we now know that the cervical collagen network is highly heterogeneous, with interweaving zones of collagen that change along the cervix from the internal to external os (Fig. 2) [6]. Further, the strength of the collagen network (and thus the tissue) depends on the degree and type of collagen crosslinks between each collagen microfibril [3,7-10] and recent studies show that the internal os has significant collagen crosslink heterogeneity compared to the external os [11]. In other words, the cervix is not homogeneous, nor are the zones as distinct as previously thought. Also, because structure relates to function, it is logical that architectural differences in the internal os compared to the external os are due to functional/physiological differences. A critical concept illuminated by these findings is that "location matters" when studying the cervix.

Related to the importance of location, previous studies suggest that the average cellular content in the cervix is about $10-15 \%$ but recent data show that the area of the internal os is significantly more cellular than the external os and contains around 50-60\% smooth muscle cells (SMCs). More intriguing is that these SMCs (i) are generally circumferentially oriented around the endocervical canal, possibly similar to a "sphincter," (Fig. 3), (ii) express contraction-associated proteins (i.e. gap junctions), and (iii) are functional (i.e. when treated with oxytocin ex vivo, they are contractile like uterine SMCs) (J. Vink, N.M. Zork, H. Feltovich, et al., unpublished data). In fact, there is a foundation for direct communication between them (via gap junctions on SMCs). These findings suggest that the uterus and cervix are less structurally and functionally distinct than previously believed. Adding to the 
complexity, a layer of epithelial cells surrounds the cervical stroma, forming an active gateway and communication pathway to the external vaginal environment $[12,13]$.

This brief overview of emerging concepts highlights three important and evolving concepts about the cervix: (i) its architectural heterogeneity means that properties of the external os are likely not generalizable to the internal os; (ii) the internal os may actually resemble a sphincter - thus "sphincter failure" may explain why the internal os dilates/funnels first in premature cervical remodeling; and (iii) the structural/physiological continuity between the cervix and uterus suggests that the cervix may play a much more active role during pregnancy than previously appreciated.

\section{Normal cervical remodeling}

Three essential elements are required for spontaneous delivery of a fetus: cervical remodeling/ripening, decidual activation, and uterine contractions (Fig. 4) [14-16]. To date, neither the way in which these elements interact nor any of their underlying molecular mechanisms have been fully elucidated. That said, the pathway to birth seems to begin and end with the cervix: human and animal studies suggest that softening begins just after conception, and the cervix obviously dilates completely just before birth.

"Cervical remodeling" is the collective term for progressive cervical change and recovery during pregnancy and involves four overlapping phases: a long, progressive softening phase; an accelerated phase of marked softening and increasing compliance near the end of pregnancy (ripening); active dilation just prior to delivery; and postpartum repair [17]. Remodeling has been studied predominantly in rodents because of inherent difficulties with obtaining tissue from pregnant women. In rodents, the softening phase is characterized by an increase in collagen solubility (decreased collagen crosslinking) [17] and a decrease in enzymes which form collagen crosslinks [18]. Recent study has also demonstrated that the ratio of immature (weaker) to mature (stronger) collagen crosslinks is decreased in early softening, suggesting that as mature crosslinked collagens decline, they are replaced by immature collagens, which increases tissue compliance [8]. In addition to collagen crosslink changes, cervical softening likely entails alterations to other matricellular proteins that modulate collagen formation and interactions between cells and the ECM (e.g. thrombospondin 2 and tenascin-C) [18].

Cervical softening is followed by ripening, and together these phases create extremely compliant tissue that easily dilates to allow for delivery of the fetus. Studies have suggested that the ripening phase is characterized by (i) increased collagen fiber diameter and spacing between fibrils as well as a shift from straight to wavy fibers, (ii) an increase in synthesis of hyaluronic acid (HA, which increases tissue hydration and thus, presumably, compliance), and (iii) an influx or activation of immune cells which release matrix metalloproteinases (MMPs, enzymes that degrade ECM) [19]. However, recent studies have shown that neither HA [20] nor immune cell activation is essential to ripening in the mouse [19]. A further complication is that the effect of the fluctuating hormonal milieu of pregnancy on remodeling remains very unclear, although it is clear that this differs between rodents and humans [19]. 
Related to this, it is important to remain mindful that even if many biochemical processes are similar between humans and rodents, there are markedly different mechanical forces at play (humans are bipeds, rodents are quadrupeds) and the species have different reproductive anatomy (humans have one uterus, mice two), which brings into question the generalizability of rodent studies. In summary, we still do not fully understand the physiology of normal cervical remodeling, particularly in humans.

\section{What is cervical insufficiency?}

Preterm birth, like term birth, requires cervical ripening, rupture of membranes, and uterine contractions. The assumed sequence, given appropriately timed activation and interaction of these pathways, is cervical remodeling/ripening $\rightarrow$ decidual activation $\rightarrow$ uterine contractions [16]. In contrast, spontaneous preterm birth (sPTB) is thought to originate mostly from factors (e.g. bleeding, uterine overdistension, infection) that cause preterm premature rupture of membranes (PPROM; decidual activation) or preterm labor (PTL; uterine contractions), which then secondarily activate cervical remodeling/ripening. In other words, the presumed sequence is decidual activation or uterine contractions $\rightarrow$ cervical remodeling/ripening [16]. Primary cervical dysfunction leading directly to sPTB is considered only a minor contributor.

The term applied to primary cervical dysfunction, "cervical insufficiency (CI)" or "cervical incompetence," represents cervical "failure" that results in midtrimester pre-/peri-viable pregnancy loss or sPTB. Its presumed cause is "weak" cervical tissue, intrinsic or acquired. However, our understanding of the pathophysiology of this condition is extremely limited. Studies implicating acquired CI (cervical surgery, e.g. conization or obstetrical injury, e.g. obstetrical laceration) conflict [21,22]. Similarly, studies attempting to identify etiologies of intrinsic CI (e.g. congenital uterine anomalies such as bicornuate uterus [21,22], genetic or tissue alterations that could contribute to an abnormal collagen/ECM/tissue formation [2329]) have been inconclusive. Even if there were sufficient evidence for acquired or intrinsic defects leading to weak cervical tissue, a bothersome question would persist: why do $50 \%$ of women with cervical insufficiency deliver at term in subsequent pregnancies? [30]

\section{Diagnosing cervical insufficiency}

Our inability to find a unifying etiology for CI is reflected in the fact that there are no diagnostic criteria for a "weak" cervix. Ultrasound findings may be suggestive; for instance, cervical "funneling" (proximal dilation of the internal os) is considered clinical evidence of CI [31]. However, although it is associated with an increased risk of sPTB, funneling does not necessarily lead to that outcome [31]. In addition, there are multiple definitions of "funneling," none of which will diagnose if the cervix is too weak to maintain a pregnancy past the midtrimester (i.e. CI). More problematic is that funneling is actually a sign of appropriate cervical remodeling; in term laboring patients, cervical change from a " $\mathrm{T}$ " shape (long/closed cervix) to a "Y", then "V", then " $U$ " is observed [31] and, in a prospective observational trial of induction of labor at term, funneling (present in $>50 \%$ of women) was a better predictor of success than Bishop score or cervical length [32]. 
Cervical shortening is even more puzzling. In obstetrical practice, a short cervix is recognized as a predictor of SPTB because of the inverse relationship between midtrimester cervical length and gestational age at delivery [33]. Unlike funneling, cervical length is easy to define and measure. Like funneling, however, cervical shortening is not diagnostic because the cervix progressively shortens in normal pregnancy, and most women with a midtrimester short cervix ( $<25 \mathrm{~mm}$ ) deliver at term. In the Preterm Prediction Study, only $27 \%$ of women with a midtrimester short cervix delivered before 37 weeks, and $<18 \%$ before 35 weeks [33] and, in a subsequent study, even a very short cervix ( $₫ 5 \mathrm{~mm}$ ) conferred only a 50\% chance of delivering before 33 weeks [34].

Even if ultrasound findings were more predictive, there are no objective tests to evaluate cervical tissue strength as a means to confirm a diagnosis of CI. Various methods have been attempted (e.g. using cervical dilators, balloons or radiographic techniques) but none have been successful [21]. In summary, we have no tests to detect biomechanical tissue changes, either actual (via cervical tissue strength testing) or presumed (via ultrasound detection of funneling or short cervix), that diagnose the cervix too weak to carry a pregnancy past the second trimester.

\section{Treating cervical insufficiency}

Our approach to treating CI reflects our lack of understanding even better than our inability to identify diagnostic criteria. Based on the presumption that acquired or intrinsic defects cause weak cervical tissue, the mainstay of treatment for CI is cerclage, a stitch around the cervix intended to keep it closed [21]. Cerclage reduces the risk of sPTB by about $30 \%$ in women with a history of SPTB and short cervix, although at least one in three of these women still delivers preterm [35]. Progesterone supplementation (intramuscular 17ahydroxyprogesterone caproate) for women with a history of SPTB is recommended by the American College of Obstetricians and Gynecologists (ACOG) [21] and the Society for Maternal-Fetal Medicine (SMFM) [36] because it reduces the risk of recurrent sPTB. The SMFM additionally supports adjunctive cerclage in those who develop cervical shortening because of a trend toward benefit [36]. Also, both societies recommend progesterone (vaginal suppositories) for risk reduction of SPTB in women with an incidental short cervix (no prior sPTB) $[22,36]$. Interestingly, cerclage does not reduce risk in women with a short cervix if they have no history of SPTB [37]. Another potential treatment is the cervical pessary, which hypothetically prevents progression of shortening/dilation by changing the angle of the cervix. However, whereas the pessary reduced risk of SPTB in one recent randomized controlled trial of women with a short cervix (with or without prior sPTB) [38], a subsequent study failed to show benefit [30].

It is unsurprising that our therapeutic armamentarium is limited and mysterious (i.e. whereas vaginal progesterone reduces SPTB risk in any woman with a short cervix, cerclage only helps those with a history of SPTB) because, in short, we do not know what we are treating. Evidence for this is provided by the Born Too Soon Preterm Prevention Analysis Group, which reported that even if all at-risk pregnancies were identified, and current interventions applied appropriately, the sPTB rate would be reduced by a disappointingly tiny 5\% [40]. 


\section{7. "Cervical insufficiency" revisited}

The above discussion illustrates that we do not know what "cervical insufficiency" really is. One fundamental problem is its imprecise definition. For example, CI is sometimes defined as a biochemical and biomechanical problem (structural "weakness" due to acquired or inherent cervical tissue defects), sometimes as a clinical problem (the event/outcome of "asymptomatic" midtrimester pregnancy loss), but usually the phrase is meant to encompass both. In contrast, PPROM has a discrete clinical definition: pre-labor rupture of membranes before 37 weeks. The definition of preterm labor (PTL) is similarly discrete and clinical: regular uterine contractions accompanied by cervical change.

CI has been defined as "painless cervical dilation after the first trimester with subsequent expulsion of the pregnancy in the second trimester, typically before 24 weeks of gestation, without contractions or labor and in the absence of other clear pathology (e.g. bleeding, infection, ruptured membranes)" [21]. The use of the word "typically" is telling because some women who are ultimately diagnosed with CI initially present with pelvic pressure, cramping, and/or vaginal discharge whereas others have a "late presentation" characterized by advanced dilation and shortening in addition to spotting, prolapsed or ruptured membranes, and/or irregular, infrequent contractions that seem inconsistent with the cervical findings [41]. In other words, CI may look exactly like PTL or PPROM - which begs the question: in the woman who presents with PPROM, PTL, and cervical dilation, which happened first - preterm activation of the decidua, the myometrium, or the cervix? This is the problem of the chicken and the egg, and, exactly like that rhetorical problem, does it matter which came first?

Ultimately, it may not matter. Cervical function was generally considered dichotomous (i.e. a cervix was either competent or incompetent) until the turn of this century, at which point it was suggested that cervical function/dysfunction is better described as a continuum [42]. More recently, a secondary analysis of data from the Preterm Prediction Study definitively demonstrated the inextricable connection between the cervix, the membranes, and the uterus: among women with $\mathrm{SPTB}$, cervical shortening occurred at the same rate whether the presenting symptom was CI, PPROM, or PTL [18]. The scope of this immense problem is underscored by the findings of a recent multinational study that identified 12 combinations ("clusters") of maternal (e.g. hypertension), fetal (e.g. anomalies), and/or placental (e.g. bleeding) conditions associated with SPTB. No precipitating factors for SPTB were found in about one in four cases [45]. This demonstrates that, despite decades of research, we still barely understand the problem of sPTB. Clearly, a new approach is needed.

\section{A new paradigm}

We propose a new paradigm rooted in the concept that the three essential elements of SPTB are inextricably intertwined such that they all lead to each other. A model based on this paradigm features the cervical contribution to SPTB (premature cervical remodeling) as equal to that of the decidua (PPROM) and myometrium (PTL) (Fig. 4). Within this model, premature cervical remodeling is simply a neutral, descriptive term that points to no cause, primary or secondary, exactly like the terms "PPROM" and "PTL". The model highlights 
the central tenet of the paradigm, namely, that these three essential elements happen in no particular order. Further, the model easily admits each of the myriad factors (some known, some yet to be discovered) that may trigger premature activation of one or more of the three essential elements, such as inflammation/infection, hemorrhage, uterine stretch, stress (i.e. activation of the maternal or fetal hypothalamic-pituitary-adrenal axis), immune dysregulation, inherent genetic alterations, behavioral/social attributes, and many others. Most importantly, this paradigm/model assigns equal weight to all three essential elements irrespective of which was activated first. Among other things, this new paradigm could unify lines of investigation and treatment that were previously divided into "sPTB due to PTL and/or PPROM" and "sPTB due to CI", streamlining research efforts [18]. In other words, this new approach has the potential to speed creative thinking about SPTB, facilitating discovery of novel interactions between the cervix, uterus, membranes, fetus, placenta, and surrounding environment (structural and molecular), and leading to novel therapeutics.

\section{The term "cervical insufficiency" is anachronistic}

A fundamental requirement of any new approach is clear terminology. Toward that end, we propose to retire the term "cervical insufficiency" and adopt the broader yet better-defined term "premature cervical remodeling". There has been a recent proposal to restore the term "precocious cervical ripening", used in the 1980s to describe a combination of features including cervical shortening, softening, funneling, and internal os dilation [46]. Although we agree with that reasoning, we prefer the more encompassing term "premature cervical remodeling" because "ripening" invokes only the late stage of cervical remodeling and it is likely that the activating events are initiated long before the ripening phase.

\section{Objective detection of "premature cervical remodeling"}

The call for a paradigm shift is much simpler than its actualization. In the case of exploring cervical remodeling as a contributor to $\mathrm{SPTB}$, success depends upon objective detection and quantification (measurement) of its various components.

\subsection{Cervical shortening/funneling}

A short cervix has a discrete clinical definition $(<25 \mathrm{~mm}$ in women with a prior sPTB or $ک 20$ $\mathrm{mm}$ in unselected women), and funneling has several, albeit relatively clear, definitions [31]. Whereas these parameters definitely belong in any model of cervical remodeling, the highly inconsistent outcomes (e.g. term vs preterm delivery) associated with various cervical lengths and degrees of funneling means that these parameters should not be used in isolation to diagnose premature cervical remodeling. Until more precise methods are available, however, a short cervix is our best indicator of premature cervical remodeling.

\subsection{Cervical softening}

The gold standard for clinical assessment of cervical softness is the "cervical consistency" component of the Bishop score, in which the cervix is pronounced "soft, medium or hard" based on (subjective) digital examination. This is remarkable given that a soft cervix (detected as early as six weeks of gestation, the "Hegar sign") has been a recognized sign of pregnancy since the 1800 s. Fortunately, however, new technologies are currently emerging 
to quantify cervical tissue softness and its relationship to the underlying collagen microstructure in pregnancy.

Compression (strain) elastography is a non-invasive (ultrasound-based) method in which gentle pressure is applied by the operator via the ultrasound transducer, or else the transducer is held still while arterial pulsations and breathing movements cause tissue movement, after which specialized software produces a color map that describes deformation of the tissue relative to neighboring areas ("strain"). Although several studies have suggested that higher cervical strain values are associated with $\mathrm{SPTB}$, the current lack of a reliable means to standardize the force applied to the tissue significantly limits the clinical usefulness of this technique $[45,46]$.

Another technique is shear wave elastography. Its main advantage over compression elastography is that measurements are relatively independent of operator force. For this method, a short-duration, high-frequency ultrasound pulse is sent into the tissue. The shear waves this creates can be monitored with typical ultrasound imaging, so that the speed of the waves can be calculated. These shear wave speeds can objectively describe tissue softness because waves travel more slowly in softer tissue. Further, they provide information about tissue microstructure because shear wave speed leads to calculation of the tissue's shear modulus (stiffness), which is dependent on fiber (i.e. collagen) orientation and organization. In an initial feasibility study, shear wave speeds detected significantly softer tissue and concomitant microstructural disorganization in human hysterectomy specimens ripened with misoprostol (a prostaglandin used clinically to produce cervical softening) compared to those that were not ripened [47]. Importantly (and unexpectedly at the time, since evidence of different cellular content at the internal versus external os did not yet exist), the shear wave speeds and other quantitative ultrasound parameters differed significantly at the internal versus the external os. In a subsequent feasibility study of women undergoing induction of labor at term, statistically significant differences in shear wave speeds were found in cervical tissue before and after ripening with misoprostol [48]. Therefore, this technique may prove to be a promising tool for objective assessment of cervical softening.

Research efforts into the pathophysiology (and related etiologies) underlying premature cervical remodeling will naturally expand as successful techniques for objective quantification of parameters such as cervical softening and collagen organization emerge.

\section{Novel approaches to studying human cervical remodeling}

Anatomically correct computer simulation models of the pregnant human pelvis, uterus and cervix are promising for studying human cervical remodeling in pregnancy. This is extremely encouraging given the limitations of rodent models and challenges of obtaining human cervical tissue in pregnancy. Recent studies utilizing these anatomically correct computer simulation models have shown that the angle of the cervix in the pelvis influences the amount of stretch exerted on the area of the internal os [45]. In addition, simulations show that the strength of the fetal membranes influences the degree of stretch at the internal os [49]. Combining these findings, it is reasonable to hypothesize that, among all women with premature cervical remodeling, those who ultimately deliver preterm also exhibit weak 
fetal membranes and/or an undesirable cervical position/angle that increases stretch at the internal os, which ultimately leads to cervical dilation and sPTB. Studies evaluating the utility of these computer models (which incorporate patient-specific tissue properties and ultrasound-derived parameters) to predict and/or identify which women with premature cervical remodeling will actually deliver preterm are currently ongoing.

\section{The future}

Solving the complex dilemma of sPTB will require an open and creative multidisciplinary collaboration, including crosstalk between experts in obstetrics, biochemistry, molecular biology, biomechanical engineering, and systems biology. Such an approach has the potential to finally uncover the pathophysiology of premature cervical remodeling, as well as PTL and PPROM, and their individual contributions to SPTB on a patient-specific level. Such a comprehensive scope may guide us to novel treatments for the multifaceted problem of sPTB.

\section{Acknowledgments}

Funding sources

The research included in this review was supported financially by the Society for Maternal Fetal Medicine/ American Association of Obstetricians \& Gynecologists Foundation Scholarship (J.V.) and NIH R21HD063031, NIH R21 HD061896, and NIH R01HD072077 from the Eunice Kennedy Shriver National Institute of Child Health and Human Development, and Intermountain Research \& Medical Foundation (H.F.).

\section{References}

1. Danforth DN. The morphology of the human cervix. Clin Obstet Gynecol. 1983; 26:7-13. [PubMed: 6839572]

2. Timmons B, Akins M, Mahendroo M. Cervical remodeling during pregnancy and parturition. Trends Endocrinol Metab. 2010; 21:353-61. [PubMed: 20172738]

3. House M, Kaplan DL, Socrate S. Relationships between mechanical properties and extracellular matrix constituents of the cervical stroma during pregnancy. Semin Perinat. 2009; 33:300-307.

4. Dubrauszky V, Schwalm H, Fleischer M. Fibre system of connective tissue in childbearing age, menopause and pregnancy. Archiv Gynakol. 1971; 210:276-92.

5. Aspden RM. Collagen organization in the cervix and its relation to mechanical function. Coll Relat Res. 1988; 8:103-12. [PubMed: 3378391]

6. Reusch LM, Feltovich H, Carlson LC, et al. Nonlinear optical microscopy and ultrasound imaging of human cervical structure. J Biomed Opt. 2013; 18:031110. [PubMed: 23412434]

7. Eyre DR, Paz MA, Gallop PM. Cross-linking in collagen and elastin. Annu Rev Biochem. 1984; 53:717-48. [PubMed: 6148038]

8. Yoshida K, Jiang H, Kim M, et al. Quantitative evaluation of collagen crosslinks and corresponding tensile mechanical properties in mouse cervical tissue during normal pregnancy. PLoS One. 2014; 9:e112391. [PubMed: 25397407]

9. Myers KM, Paskaleva AP, House M, Socrate S. Mechanical and biochemical properties of human cervical tissue. Acta Biomaterials. 2008; 4:104-16.

10. Myers K, Socrate S, Tzeranis D, House M. Changes in the biochemical constituents and morphologic appearance of the human cervical stroma during pregnancy. Eur J Obstet Gynecol Reprod Biol. 2009; 144(Suppl 1):S82-S89. [PubMed: 19303693]

11. Zork NM, Myers KM, Yoshida K, et al. A systematic evaluation of collagen cross-links in the human cervix. Am J Obstet Gynecol. 2015; 212:321.e1-8. [PubMed: 25281365] 
12. Elovitz MA, Brown AG, Anton L, Gilstrop M, Heiser L, Bastek J. Distinct cervical microRNA profiles are present in women destined to have a preterm birth. Am J Obstet Gynecol. 2014; 210:221.e1-11. [PubMed: 24565431]

13. Nold C, Anton L, Brown A, Elovitz M. Inflammation promotes a cytokine response and disrupts the cervical epithelial barrier: a possible mechanism of premature cervical remodeling and preterm birth. Am J Obstet Gynecol. 2012; 206:208.e1-7. [PubMed: 22285171]

14. Rubens CE, Sadovsky Y, Muglia L, Gravett MG, Lackritz E, Gravett C. Prevention of preterm birth: harnessing science to address the global epidemic. Nov 12.2014 6(262):262sr5. www.ScienceTranslationalMedicine.org.

15. Romero R, Yeo L, Chaemsaithong P, Chaiworapongsa T, Hassan SS. Progesterone to prevent spontaneous preterm birth. Semin Fetal Matern Med. 2014; 19:15-26.

16. Iams JD, Cebrik D, Lynch C, Behrendt N, Das A. The rate of cervical change and the phenotype of spontaneous preterm birth. Am J Obstet Gynecol. 2011; 205:130.e1-6. [PubMed: 22088837]

17. Read CP, Word RA, Ruscheinsky MA, Timmons BC, Mahendroo MS. Cervical remodeling during pregnancy and parturition: molecular characterization of the softening phase in mice. Reproduction. 2007; 134:327-340. [PubMed: 17660242]

18. Akins ML, Luby-Phelps K, Bank RA, Mahendroo M. Cervical softening during pregnancy: regulated changes in collagen cross-linking and composition of matricellular proteins in the mouse. Biol Reprod. 2011; 84:1053-62. [PubMed: 21248285]

19. Mahendroo M. Cervical remodeling in term and preterm birth: insights from an animal model. Reproduction. 2012; 143:429-38. [PubMed: 22344465]

20. Akgul Y, Word RA, Ensign LM, et al. Hyaluronan in cervical epithelia protects against infectionmediated preterm birth. J Clin Invest. 2014; 124:5481-5489. [PubMed: 25384213]

21. Committee on Practice Bulletins - Obstetrics, American College of Obstetricians and Gynecologists. Practice Bulletin No. 142 Cerclage for the management of cervical insufficiency. Obstet Gynecol. 2014; 123(2 Pt 1):372-9. [PubMed: 24451674]

22. Committee on Practice Bulletins - Obstetrics, American College of Obstetricians and Gynecologists. Practice Bulletin no 130: Prediction and prevention of preterm birth. Obstet Gynecol. 2012; 120:964-73. [PubMed: 22996126]

23. Warren JE, Silver RM, Dalton J, Nelson LT, Branch DW, Porter TF. Collagen 1A1 and transforming growth factor- $\beta$ polymorphisms in women with cervical insufficiency. Obstet Gynecol. 2007; 110:619-24. [PubMed: 17766609]

24. Warren JE, Nelson LM, Stoddard GJ, Esplin MS, Varner MW, Silver RM. Polymorphisms in the promoter region of the interleukin-10 (IL-10) gene in women with cervical insufficiency. Am J Obstet Gynecol. 2009; 201:372-37. [PubMed: 19591971]

25. Petersen LK, Uldbjerg N. Cervical collagen in non-pregnant women with previous cervical incompetence. Eur J Obstet Gynecol Reprod Biol. 1996; 67:41-5. [PubMed: 8789748]

26. Schlembach D, MacKay L, Shi L, Maner WL, Garfield RE, Maul H. Cervical ripening and insufficiency: from biochemical and molecular studies to in vivo clinical examination. Eur J Obstet Gynecol Reprod Biol. 2009; 144:S70-S76. [PubMed: 19303692]

27. Leppert PC, Yu SY, Keller S, Cerreta J, Mandl I. Decreased elastic fibers and desmosine content in incompetent cervix. Am J Obstet Gynecol. 1987; 157:1134-9. [PubMed: 3688067]

28. Roddick JWJ, Buckingham JC, Danforth DN. The muscular cervix - a cause of incompetency in pregnancy. Obstet Gynecol. 1961; 17:562-5. [PubMed: 13742503]

29. Buckingham JC, Buethe RAJ, Danforth DN. Collagen-muscle ratio in clinically normal and clinically incompetent cervicis. Am J Obstet Gynecol. 1965; 91:232-7. [PubMed: 14258025]

30. Edlow AG, Srinivas SK, Elovitz MA. Second-trimester loss and subsequent pregnancy outcomes: what is the real risk? Am J Obstet Gynecol. 2007; 197:581.e1-6. [PubMed: 18060941]

31. Mancuso MS, Szychowski JM, Owen J, et al. Vaginal Ultrasound Trial Consortium. Cervical funneling: effect on gestational length and ultrasound-indicated cerclage in high-risk women. Am J Obstet Gynecol. 2010; 203:259.e1-5. [PubMed: 20816149]

32. Chung SH, Kong MK, Kim EH, Han SW. Sonographically accessed funneling of the uterine cervix as a predictor of successful labor induction. Obstet Gynecol Sci. 2015; 58:188-95. [PubMed: 26023667] 
33. Iams JD, Goldenberg RL, Meis PJ, et al. The length of the cervix and the risk of spontaneous premature delivery. Am J Obstet Gynecol. 1996; 334:567-73.

34. Hassan SS, Romero R, Berry SM, et al. Patients with an ultrasonographic cervical length $<$ or $=$ $15 \mathrm{~mm}$ have nearly a $50 \%$ risk of early spontaneous preterm delivery. Am J Obstet Gynecol. 2000; 182:1458-67. [PubMed: 10871466]

35. Berghella V, Rafael T, Szychowski J, Rust OA, Owen J. Cerclage for short cervix on ultrasound in singleton gestations with prior preterm birth: meta-analysis of trials using individual patient-level data. Obstet Gynecol. 2011; 117:663-71. [PubMed: 21446209]

36. Society for Maternal-Fetal Medicine Publications Committee, with assistance of Vincenzo Berghella. Progesterone and preterm birth prevention: translating clinical trials data into clinical practice. Am J Obstet Gynecol. 2012; 206:56-86. [PubMed: 21924397]

37. Berghella V, Odibo AO, To MS, Rust OA, Althuisias SM. Cerclage for short cervix on ultrasonography: meta-analysis of trials using individual patient-level data. Obstet Gynecol. 2005; 106:181-9. [PubMed: 15994635]

38. Goya M, Pratcorona L, Merced C, et al. Pesario Cervical para Evitar Prematurdad (PECEP) Trial Group. Cervical pessary in pregnant women with a short cervix (PECEP): an open-label randomized controlled trial. Lancet. 2012; 379(9828):180-6.

39. Hui SY, Chor CM, Lau TK, Lao TK, Leung TY. Cerclage pessary for preventing preterm birth in women with a singleton pregnancy and a short cervix at 20 to 24 weeks: a randomized controlled trial. Am J Perinatol. 2013; 30:283-8. [PubMed: 22875662]

40. Chang HH, Larson J, Blencowe H, et al. Born Too Soon Preterm Prevention Analysis Group. Preventing preterm births: analysis of trends and potential reductions with interventions in 39 countries with very high human development index. Lancet. 2013; 381:223-34. [PubMed: 23158883]

41. Berghella, V. [last accessed August 11, 2015] Cervical insufficiency. UpToDate.com

42. Owen J, Iams JD, Hauth JC. Vaginal sonography and cervical incompetence. Am J Obstet Gynecol. 2003; 188:586-96. [PubMed: 12592276]

43. Barros FC, Papageorghiou AT, Victoria CG, et al. The distribution of clinical phenotypes of preterm birth syndrome: implications for prevention. JAMA Pediatr. 2015; 169:220-9. [PubMed: 25561016]

44. Li Q, Reeves M, Owen J, Keith LG. Precocious cervical ripening as a screening target to predict spontaneous preterm delivery among asymptomatic singleton pregnancies: a systematic review. Am J Obstet Gynecol. 2015; 212:145-56. [PubMed: 25017411]

45. Myers KM, Feltovich H, Mazza E, et al. The mechanical role of the cervix in pregnancy. J Biomech. 2015; 48:1511-23. [PubMed: 25841293]

46. Hee L. Overview of the methods available for biomechanical testing of the uterine cervix in vivo. Acta Obstet Gynecol Scand. 2014; 93:1219-37. [PubMed: 25174701]

47. Carlson LC, Feltovich H, Palmeri ML, Dahl JJ, Munoz del Rio A, Hall TJ. Shear wave speed estimation in the human uterine cervix. Ultrasound Obstet Gynecol. 2014; 43:452-8. [PubMed: 23836486]

48. Carlson LC, Romero ST, Palmeri ML, et al. Changes in shear wave speed pre and post induction of labor: a feasibility study. Ultrasound Obstet Gynecol. 2015; 46:93-8. [PubMed: 25200374]

49. Fernandez M, House M, Jambawalikar S, et al. Investigating the mechanical function of the cervix during pregnancy using finite element models derived from high-resolution 3D MRI. Comput Methods Biomech Biomed Engng. 2015 May 13.:1-14. 


\section{Practice points}

- Cervical heterogeneity means that sampling (study) location matters; in other words, molecular events and biomechanical properties of the internal os (where premature cervical remodeling seems to manifest primarily) are unlikely to be the same as the external os (which so far has received the greatest amount of research attention).

- The cellular content of the cervix is much greater than $10-15 \%$; the area of the internal os contains $50-60 \%$ smooth muscle cells, arranged similar to a sphincter.

- The uterus and cervix are apparently not structurally distinct; the lower uterus and upper cervix both contain a high proportion of smooth muscle cells, and the gap junctions between them makes direct communication possible and even likely.

- A paradigm shift in which the ill-defined term "cervical insufficiency" is retired and the descriptive, singularly defined term "premature cervical remodeling" is adopted could lead to a model of SPTB in which complex interactions between the cervix, uterus, membranes, fetus, placenta, and surrounding (structural and molecular) environment are appropriately honored. This, in turn, could facilitate novel, targeted, and truly effective treatment options. 


\section{Research directions}

- Specifically define the function of the internal os during normal and premature cervical remodeling.

- Develop non-invasive tools to pinpoint premature cervical remodeling in order to facilitate targeted molecular studies.

- Elucidate how mechanotransduction influences cervical tissue remodeling (i.e. the effect on remodeling of mechanical forces such as stretch).

- Investigate the effect of the surrounding environment (e.g. the vaginal microbiome) on normal and premature cervical remodeling.

- Establish how current therapies (e.g. progesterone, cerclage, pessary) may prevent or delay premature cervical remodeling.

- Explore combinations of putative pathways to SPTB in order to develop novel therapies. 


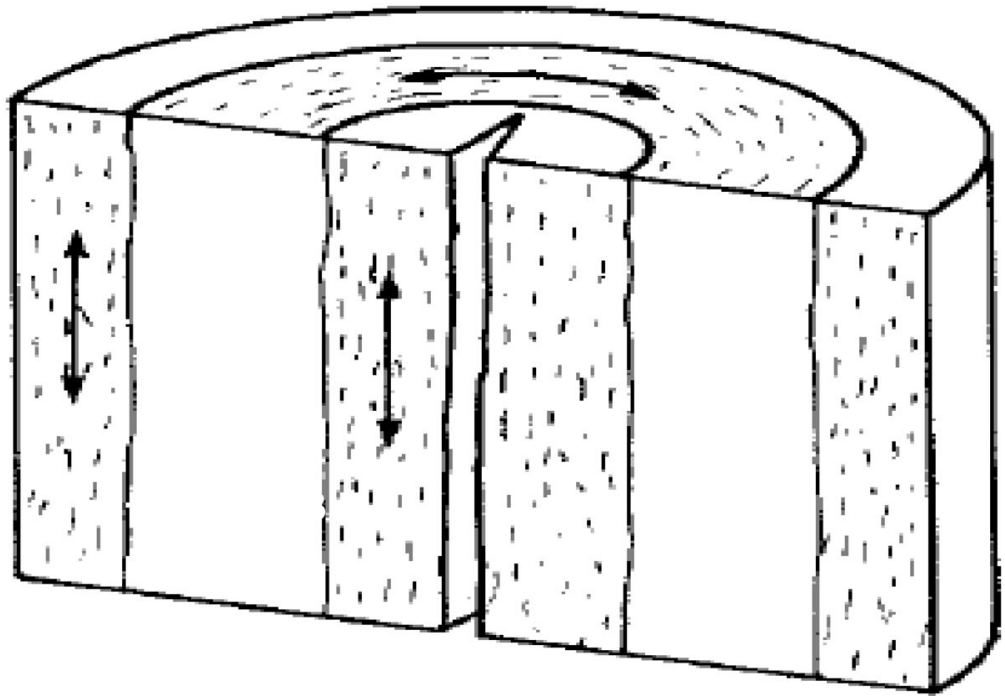

Fig. 1.

The three-zone theory of the cervical collagen network: inner and outer zones contain mostly collagen fibers that are oriented parallel to the endocervical canal, and a middle zone contains collagen fibers that are circumferentially oriented around the endocervical canal. 


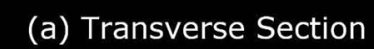

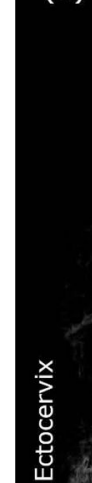

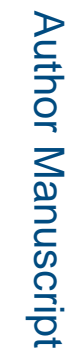

Fig. 2. (b) Longitudinal Section

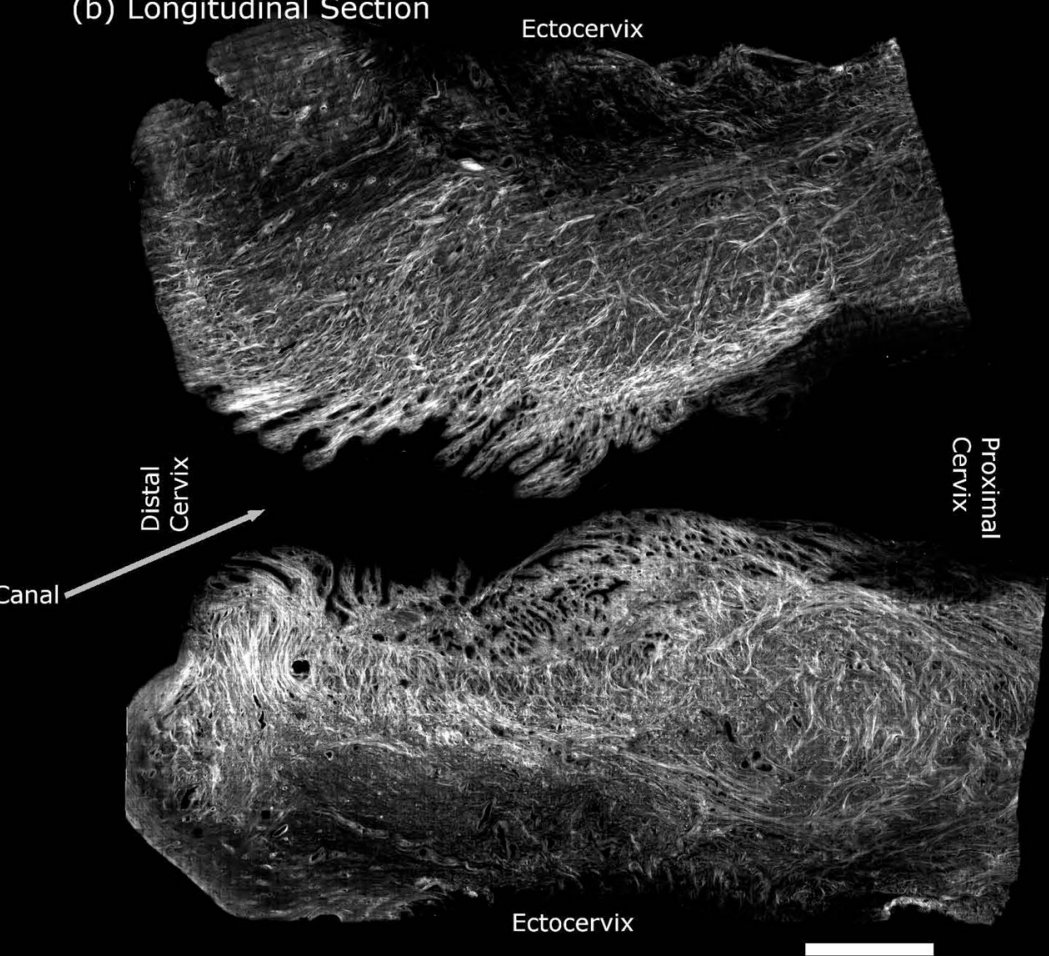

$5 \mathbf{m m}$

Second harmonic generation microscopy image of human cervical tissue showing the complex collagen network. The right side of the image shows longitudinal sections taken from the endocervical canal, with the distal cervix on the left and proximal on the right. The left side of the image shows transverse sections taken from midway between the distal and proximal ends of the cervix (upper: anterior cervix; lower: posterior cervix). 

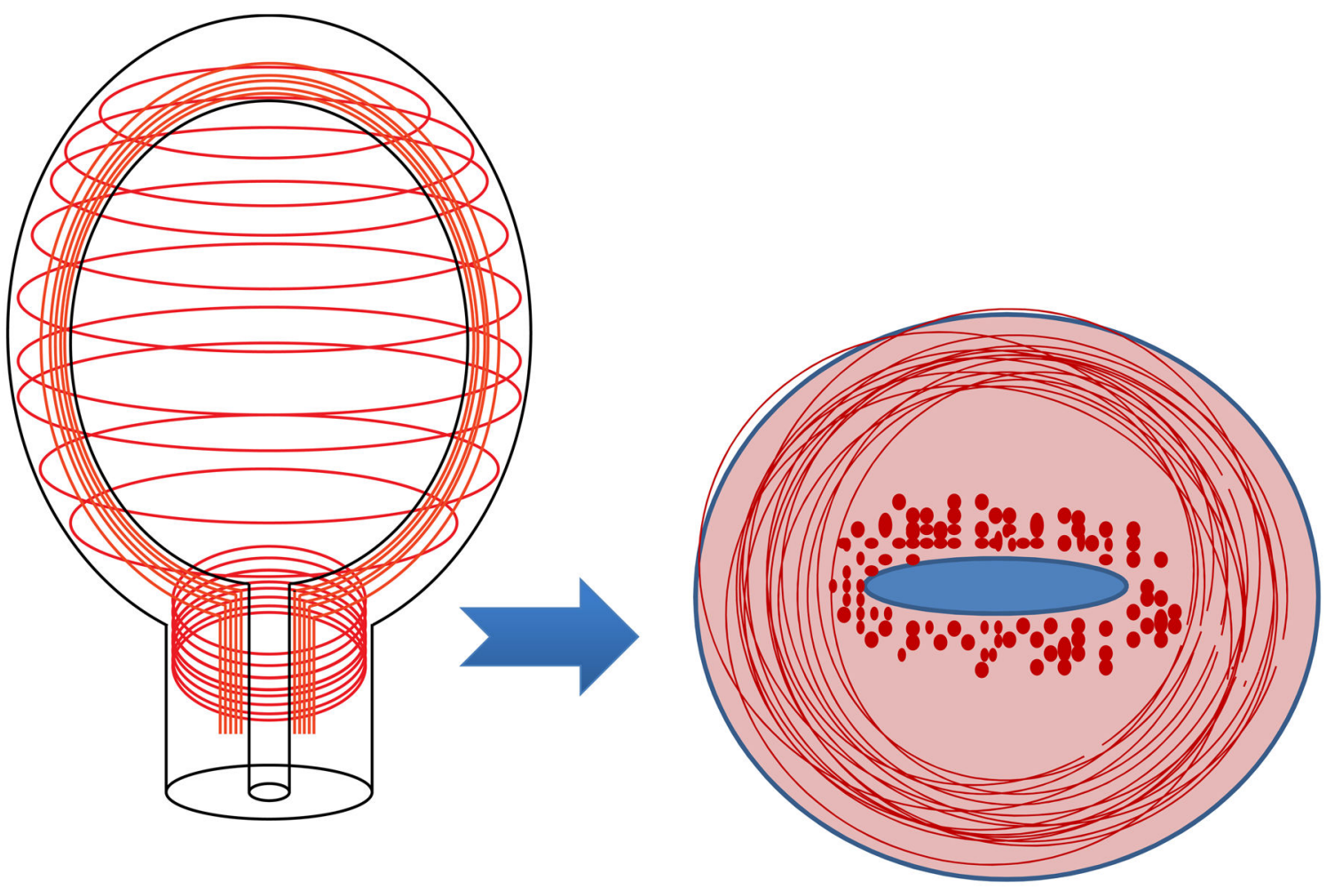

Fig. 3.

The emerging view of uterine and cervical smooth muscle cell (SMC) architecture. The area of the internal os contains circumferential SMCs that are contractile and resemble a "sphincter." These SMCs express gap junctions which are a means to directly communicate with uterine SMCs. 


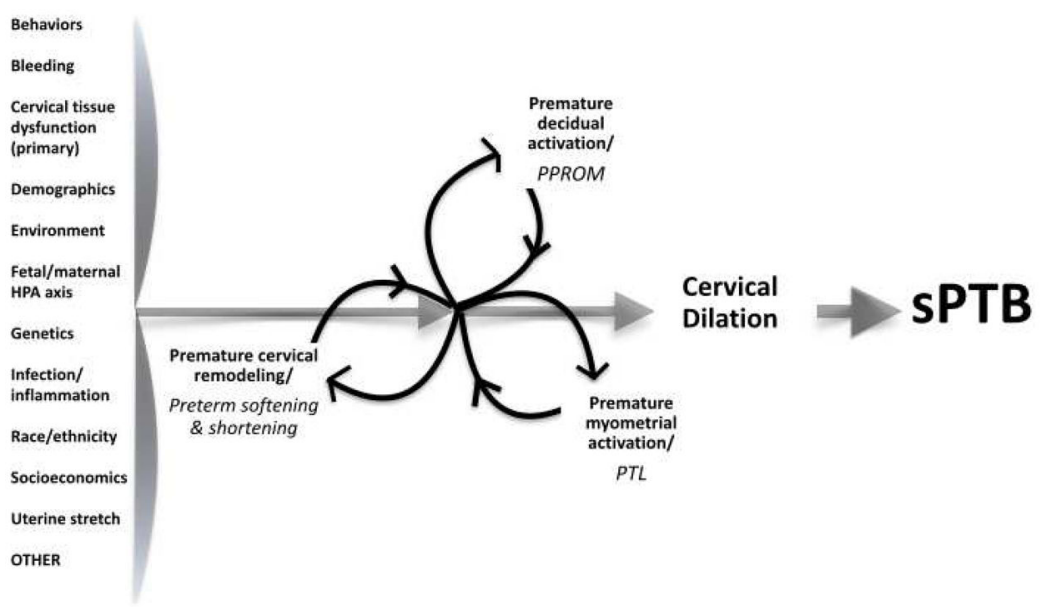

Fig. 4.

Cervical remodeling. HPA axis, hypothalamic-pituitary-adrenal axis; PPROM, preterm premature rupture of membranes; PTL, preterm labor; $\mathrm{sPTB}$, spontaneous preterm birth. 